University of Nebraska - Lincoln

DigitalCommons@University of Nebraska - Lincoln

June 2006

\title{
Selenoproteins and selenoproteomes
}

Vadim N. Gladyshev

University of Nebraska-Lincoln, vgladyshev@rics.bwh.harvard.edu

Follow this and additional works at: https://digitalcommons.unl.edu/biochemgladyshev

Part of the Biochemistry, Biophysics, and Structural Biology Commons

Gladyshev, Vadim N., "Selenoproteins and selenoproteomes" (2006). Vadim Gladyshev Publications. 36. https://digitalcommons.unl.edu/biochemgladyshev/36

This Article is brought to you for free and open access by the Biochemistry, Department of at DigitalCommons@University of Nebraska - Lincoln. It has been accepted for inclusion in Vadim Gladyshev Publications by an authorized administrator of DigitalCommons@University of Nebraska - Lincoln. 
Published in Selenium: Its molecular biology and role in human health, Second Edition, edited by Dolph L. Hatfield, Marla J. Berry, and Vadim N. Gladyshev. Springer Science+Business Media LLC, 2006. Pages 99-114. Copyright (C) 2006 Springer Science+Business Media LLC. Used by permission.

\title{
CHAPTER 9 \\ Selenoproteins and selenoproteomes
}

\author{
Vadim N. Gladyshev \\ Department of Biochemistry, University of Nebraska-Lincoln
}

\begin{abstract}
Summary: In the past several years, progress in genome sequencing and development of specialized bioinformatics tools allowed efficient identification of selenocysteine-containing proteins encoded in completely sequenced genomes. Information is currently available on selenoproteomes from a variety of organisms, including humans, which contain 25 known selenoprotein genes. This review provides basic information about mammalian selenoproteins and other known selenoprotein families. Analysis of full sets of selenoproteins in organisms provides exciting avenues for examining selenoprotein evolution and dependence of organisms on the trace element selenium and allows linking selenoproteins with specific biological and biomedical effects of dietary selenium.
\end{abstract}

\section{Introduction}

Selenium occurs in selenoproteins in two known forms, In several bacterial selenium-containing molybdoproteins, such as nicotinic acid hydroxylase and xanthine dehydrogenase, it is present in the form of a labile cofactor that contains a Se-Mo bond that is directly involved in catalysis [1-4]. Neither the exact chemical form of selenium in this cofactor nor its biosynthetic pathway are known.

However, by far the major form of selenium in proteins is selenocysteine (Sec), the $21^{\text {st }}$ amino acid in the genetic code. It is encoded by TGA and has been found in all three major lines of descent (bacteria, archaea and eukaryotes). Research in the past five years strengthened the idea that the essential role of selenium in biology, as well as its beneficial roles in human health, are largely due to its presence in proteins in the form of Sec. In contrast to other amino acids found in proteins, Sec is typically utilized only when it is required for protein function. Accordingly, it is normally a key functional (catalytic) group in proteins, and essentially all selenoproteins with known functions directly use $\mathrm{Sec}$ in redox catalysis.

\section{Bioinformatics tools for selenoprotein identification}

Historically, selenoproteins have been identified by following the presence of selenium in protein fractions during isolation. Sec-containing proteins can 
be metabolically labeled with ${ }^{75} \mathrm{Se}$, a convenient y-emitter that remains covalently bound in proteins during SDS-PAGE and can be visualized on gels and membranes using a PhosphorImager. Using this technique, a number of proteins were identified in both prokaryotes and eukaryotes [5-8]. However, it has become apparent that the applicability of this technique is limited to the most abundant proteins, whereas less abundant selenoproteins and those characterized by unique expression patterns could not be identified by this method.

Remarkable progress in genome sequencing and other large-scale sequencing projects provided an attractive and mostly untapped resource that could be utilized for selenoprotein discovery. All selenoprotein genes have two characteristic features: a Sec-encoding TGA codon and a Sec insertion sequence (SECIS) element. The TGA triplets that code for Sec do not provide sufficient information at the nucleotide sequence level to identify them computationally. However, SECIS elements are amenable to these techniques as these structures are highly specific for selenoprotein genes, have conserved segments and possess a sufficiently complex secondary structure (Chapters 57). Therefore, initial bioinformatics analyses focused on SECIS elements, and selenoprotein discovery followed a simple strategy: 1) finding candidate SECIS elements; 2) analyzing upstream regions to identify coding regions; and 3) testing candidate selenoproteins for the presence of selenium by metabolically labeling cells with ${ }^{75} \mathrm{Se}$. The first selenoproteins identified using this technique were selenoproteins $\mathrm{R}$ (now known as methionine-R-sulfoxide reductase), $\mathrm{N}$ and $\mathrm{T}[9,10]$.

This strategy was initially limited to cDNA sequences, but, following improvements in the computational description of SECIS elements and selenoprotein genes $[\mathrm{II}]$, the searches were later carried out on entire genomes characterized by small-to-moderate sizes $[12,13]$. To analyze larger genomes, a pair of closely related genomes was used (e.g., human and mouse genomes), which were searched for conserved pairs of SECIS elements that belonged to selenoprotein orthologs in these organisms [14].

In parallel, bioinformatics tools were developed that screened for coding function of TGA codons by analyzing sequences downstream of TGA [13]. The searches were also greatly aided by the observation that most selenoprotein genes have homologs, in which Sec is replaced with Cys. As a result, a strategy was developed wherein large protein databases (e.g., NCBI non-redundant protein database) could be searched against large nucleotide sequence databases that are known to contain selenoprotein genes (e.g., genomes of organisms containing Sec insertion machinery genes) [14-16]. These searches were designed to identify TGA-containing nucleotide sequences which when translated align with Cys-containing protein sequences from the protein database such that the conserved Cys residues align with translated TGA codons and these pairs are flanked by conserved sequences. In a further extension of this approach, computational gene predictions were carried out on pairs of genomes (at the appropriate phylogenetic distance) to identify TGA to TGA and TGA to Cys codon alignments embedded in conserved stretches of sequence. This approach has also lead to the discovery of new selenoprotein families.
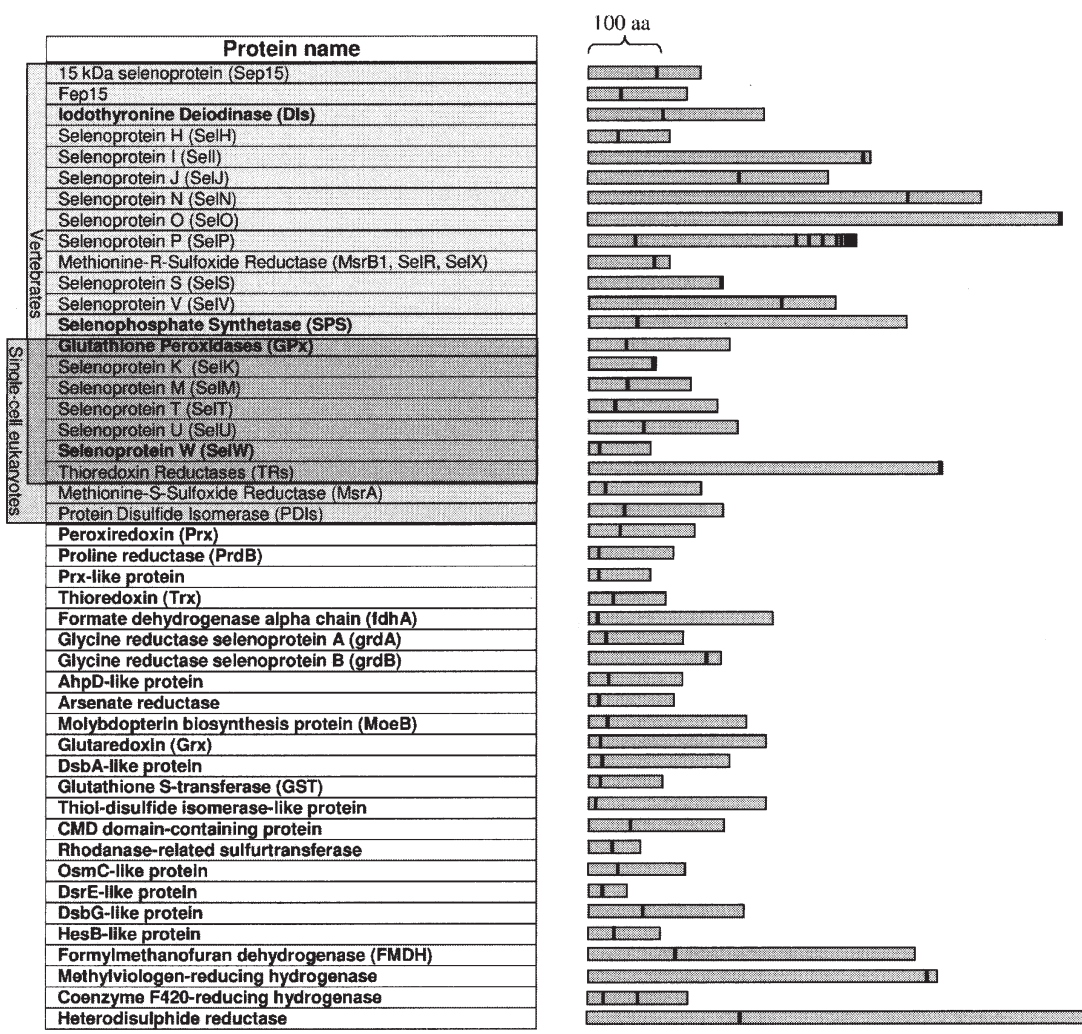

Figure 1. Selenoprotein families. Selenoproteins that occur in vertebrates or single celled eukaryotes are highlighted by shaded boxes, and Selenoproteins found in prokaryotes are shown in bold. On the right, relative sizes of Selenoproteins are shown (relative to a 100 amino acid scale) and the location of Sec within protein sequence is shown by a black line.

Thus, although searches for SECIS elements could efficiently guide computational selenoprotein predictions, the alternative methods were possible. The Sec/Cys and Sec/Sec homology approaches were completely independent of the searches for SECIS elements. Importantly, both SECIS-based and 
SECIS-independent algorithms identify similar sets of selenoprotein genes in various genomes, arguing that both tools show excellent performance and that all or almost all selenoproteins could be identified by these programs in completely sequenced genomes.

\section{Selenoproteins}

In the past five years, numerous papers reported on novel selenoprotein genes. Figure 1 lists currently known selenoprotein families. More than half of the selenoproteins in this figure are new (e.g., have been discovered after publication of the previous edition of this book), and essentially all of them were discovered through bioinformatics approaches. Some selenoproteins were named differently by various laboratories, sometimes causing confusion in regard to the number and identity of selenoproteins. Below, we briefly describe currently known selenoproteins, with the focus on selenoproteins found in mammals. More detailed information on selected selenoproteins can be found in various chapters in this book.

\section{Mammalian selenoproteins}

Glutathione peroxidases. Glutathione peroxidase 1 (GPx1) is the first known animal selenoprotein [17] and also perhaps the best studied (see Chapters 1416). It is a highly efficient antioxidant enzyme that catalyzes glutathione-dependent hydroperoxide reduction. Mammals contain eight glutathione peroxidase homologs, of which five are selenoproteins, including GPx1 (also known as cGPx), GPx2 (also known as GI-GPx), GPx3 (also known as pGPx), GPx4 (also known as PHGPx) and GPx6. Of these, only GPx4 is known to be essential during embryogenesis in mammals [18]. m addition to its antioxidant function, this protein serves a structural role in mature sperm and was implicated in site-specific disulfide bond formation [19]. Questions remain in regard to the function of all Sec-containing glutathione peroxidases. Recently, selenoprotein GPx homologs were detected in single-celled eukaryotes and bacteria.

Thyroid hormone deiodinases. Mammals have three deiodinases (DI1, DI2 and DI3) which activate or inactivate thyroid hormones by reductive deiodination. These selenoproteins are reviewed in detail in Chapter 19. It was thought that deiodinases evolved in animals, but recently, bacterial homologs of these proteins were found [16].

Thioredoxin reductases. Mammalian thioredoxin reductase (TR) was known for decades, but only recently was it discovered that these enzymes are selenoproteins (see Chapters 17 and 18). This family of proteins was the first in which Sec was found to be located at the penultimate C-terminus $[6,20]$, and we now know that $\mathrm{Sec}$ in these proteins is part of the additional active site that is itself a substrate for the N-terminal thiol-disulfide active site [21-23].
Mammals contain three thioredoxin reductases, and all three are selenoproteins [24]; therefore the entire thioredoxin system in mammals is dependent on selenium. TR1 (TrxR1, TxnRd1) is a cytosolic protein. Its main function is to control the reduced state of thioredoxin. However, it exhibits broad substrate specificity [25] and occurs in the form of at least six isoforms generated by alternative splicing that differ in their N-terminal sequences [26-28]. Thioredoxin/glutathione reductase (TGR, also known as TR2 and TrxR3) is a protein that, compared to other animal thioredoxin reductases, has an additional N-terminal glutaredoxin (Grx) domain [22]. TGR can catalyze many reactions specific for thioredoxin and glutathione systems. This protein was recently implicated in the formation/isomerization of disulfide bonds during sperm maturation [29]. TR3 (also known as TrxR2) is a mitochondrial protein, whose function is likely to reduce mitochondrial thioredoxin and glutaredoxin 2. TR1 and TR3 are essential proteins in mammals [30,31].

Methionine-R-sulfoxide reductase 1 (MsrB1). MsrB1 was initially identified using bioinformatics approaches as Selenoprotein R [9] and Selenoprotein $X[10]$, and later was shown to catalyze stereospecific reduction of oxidized methionine residues in proteins with thioredoxin as reductant (Chapter 11) [32]. In mammals, there are two additional MsrBs (MsrB2 and MsrB3); however, these contain Cys in the active site and reside in mitochondria and/ or endoplasmic reticulum [33]. MsrB1 is located in the cytosol and nucleus and is the most active MsrB in mammals [34].

$15 \mathrm{kDa}$ Selenoprotein (Sep15). Sep15 is a mammalian protein, but its homologs are found in other eukaryotes (mostly in animals) (Chapter 13) [7]. It resides in the endoplasmic reticulum where it binds UDP-glucose:glycoprotein glucosyltransferase, a sensor of protein folding [35]. Recent structural analyses revealed its redox function. Sep15 is characterized by the thioredoxin-like fold and is implicated in the cancer prevention effect of dietary selenium [36,37].

Selenophosphate synthetase 2 (SPS2). By analogy to bacterial selenophosphate synthetase SelD [38], SPS2 is thought to synthesize selenophosphate, a selenium donor compound (Chapter 4) [39]. SPS2 homologs are found in all three major domains of life.

Selenoprotein P (SelP). SelP is the only Selenoprotein with multiple Sec residues (Chapter 10) [40]. For example, human SelP has $10 \mathrm{Sec}$ and zebrafish SelPa has $17 \mathrm{Sec}$ residues. However, some organisms also have smaller SelPs (e.g., zebrafish SelPb contains a single Sec) [41]. SelP is the major plasma Selenoprotein, which is synthesized in the liver and delivers selenium to certain other organs and tissues $[42,43]$. However, brain appears to synthesize its own pool of SelP (see Chapter 21).

Selenoprotein W (SelW). SelW is the smallest mammalian Selenoprotein (Chapter 12) [44]. Its function is not known. It was thought to be specific to 
animals, but recently SelW homologs were identified in lower eukaryotes and bacteria [16].

Selenoprotein V (SelV). SelV has a C-terminal SelW homology domain and a larger N-terminal sequence of unknown function [14]. This protein is expressed exclusively in testes. Its function is not known.

Selenoprotein T (SelT). SelT is a small selenoprotein with a putative Nterminal redox motif [9]. Its function is not known.

Selenoprotein M (SelM). SelM is a distant homolog of Sepl5 and also has a thioredoxin-like fold and a predicted redox motif $[37,45]$. SelM resides in the endoplasmic reticulum. Its function is not known.

Selenoprotein $\mathrm{H}(\mathrm{SelH})$. This small selenoprotein of unknown function with a predicted redox motif was first identified as BthD in flies [12,14].

Selenoprotein $\mathrm{O}(\mathrm{SelO})$. SelO is a widely distributed protein that has homologs in animals, bacteria, yeast and plants, but the function of any of these proteins is not known [14]. Only vertebrate homologs of SelO have Sec, which is located in the C-terminal penultimate position. In SelO homologs from other organisms. Sec is replaced with Cys.

Selenoprotein K (SelK). SelK is unusual among mammalian selenoproteins in that it does not have a pronounced secondary structure [14]. This small selenoprotein contains a single transmembrane helix in the N-terminal sequence that targets this protein to the plasma membrane. SelK homologs can be detected in many eukaryotes, but no information is available on the function of this protein.

Selenoprotein S (SelS). Like SelK, SelS has Sec in the C-terminal sequence and a single transmembrane region at the N-terminus [14]. Recent studies revealed its role in retrotranslocation of misfolded proteins from the endoplasmic reticulum of mammalian cells to the cytosol, where these proteins are further degraded [46]. SelS binds Berlin 1, an endoplasmic reticulum protein also involved in protein retrotranslocation. In addition, SelS was implicated in inflammation and immune response [47].

Selenoprotein N (SelN). One of the first selenoprotein discovered through bioinformatics approaches [10], SelN remains a selenoprotein of unknown function. This protein was implicated in the role of selenium in muscle function [48].

Selenoprotein I (Sell). Sell is a recently evolved selenoprotein specific to vertebrates [14]. This predicted membrane selenoprotein has no known function.

\section{Other eukaryotic selenoproteins}

Methionine-S-sulfoxide reductase (MsrA). MsrA is a widely distributed protein family, whose function is to repair methionine residues in proteins [49]. MsrA catalyzes a stereospecific reduction of methionine-S-sulfoxides with thioredoxin. Only one selenoprotein MsrA was described, which is present in Chlamydomonas, green algae [50]. All other known MsrAs are Cys-contain- ing proteins. MsrA should not be confused with MsrB [33]. Both MsrA and MsrB are methionine sulfoxide reductases, but they have no sequence homology and catalyze complementary reactions using different diastereomers of methionine sulfoxide.

Protein disulfide isomerase (PDI). Selenoprotein PDI is narrowly distributed in eukaryotes [51], whereas Cys-containing PDI is an essential protein involved in formation of disulfide bonds in the endoplasmic reticulum of eukaryotic cells.

Selenoprotein U (SelU). SelU is found in the selenoprotein form in fish and several other marine organisms, but in mammals, all three SelU homologs are Cys-containing proteins [52]. SelU function is not known.

Selenoprotein J (SelJ). SelJ has been described in fish and sea urchin, with Cys homologs only found in cnidarians. This selenoprotein shows similarity to the jellyfish J1-crystallins and together with these proteins defines a unique subfamily within the larger family of ADP-ribosylation enzymes [53]. SelJ function is not known.

Fish $15 \mathrm{kDa}$ selenoprotein (Fep15). Fep15 is homologous to mammalian Sepl5 and SelM, but could only be detected in fish [54]. Fepl5 resides in the endoplasmic reticulum and possibly in Golgi. Its function is not known.

Plasmodium selenoproteins Sel1, Sel2, Sel3, and Sel4. The four Plasmodium selenoproteins show no detectable homology to any other proteins [55]. Sell and Sel4 have Sec in the C-terminal regions, similar to animal SelK and SelS. Functions of these proteins are not known.

\section{Prokaryotic selenoproteins}

Several selenoproteins, including selenophosphate synthetase, deiodinase homologs, glutathione peroxidase and SelW, occur in both prokaryotes and eukaryotes. These selenoproteins have already been discussed above. Other prokaryotic selenoproteins are discussed below.

Formate dehydrogenase (FDH). This is the most abundant and widespread prokaryotic selenoprotein [56]. Sec in this protein is coordinated to molybdenum and directly involved in the oxidation of formate to carbon dioxide $[57,58]$. In many bacteria, FDH is the only selenoprotein, which may be responsible for maintaining the Sec trait in these organisms [59].

Hydrogenase. Several hydrogenases are known that contain Sec. In these proteins. Sec is bound to nickel and is directly involved in catalysis [60]. Two different hydrogenase subunits may contain Sec, including one which may have two Sec residues [61].

Formylmethanofuran dehydrogenase (FMDH). FMDH is a distant homo$\log$ of FDH and catalyzes a similar reaction (with formylmethanofuran as the substrate) [62]. As in FDH, Sec in FMDH is coordinated to molybdenum in the enzyme active site. 
Selenoprotein A (GrdA). GrdA is a selenoprotein component of a multiprotein glycine reductase complex in certain bacteria [63]. This is the only known prokaryotic selenoprotein for which no Cys homologs can be detected [38].

Selenoprotein B (GrdB). GrdB is a selenoprotein component of multiprotein complexes involved in the reduction of glycine, sarcosine, betaine and other substrates [64-66]. Known GrdB proteins are substrate-specific and bind a single GrdA.

Peroxiredoxin (Prx). Peroxiredoxins are abundant Cys-containing proteins that are present in essentially all organisms. Some bacteria contain Sec-containing Prxs [67]. However, these selenoproteins have not been functionally characterized.

Thioredoxin (Trx). Trx is the major intracellular protein disulfide reductant. It occurs in all organisms and is often an essential protein. Some bacterial Trx homologs are selenoproteins $[15,16]$.

Glutaredoxin (Grx). Like Trx, it is a well studied protein disulfide oxidoreductase. Grx function is dependent on glutathione. Some bacterial Grxs are selenoproteins $[15,16]$.

HesB-like. This distant homolog of HesB proteins (also known as IscA) is a selenoprotein present in certain archaea and bacteria [15]. HesB/IscA proteins are involved in iron-sulfur cluster biosynthesis, but the function of their selenoprotein homolog has not been characterized.

Additional prokaryotic selenoproteins. Additional selenoproteins are listed in Figure 1. Most of these proteins are homologs of thiol-dependent oxidoreductases, in which the catalytic Cys is replaced with Sec $[15,16]$.

\section{Selenoprotein functions}

From the brief description of selenoprotein functions, it is apparent that selenoproteins for which functions are known are redox proteins. In these proteins, Sec is the catalytic residue that is employed because of its strong nuclephilicity and low pKa $[38,68]$. Sec reversibly changes its redox state during catalysis. Functions of many selenoproteins, particularly those found in vertebrates, are not known. However, by analogy to proteins with known functions, it may be expected that the majority of these uncharacterized selenoproteins are also redox proteins.

All selenoproteins may be loosely clustered into three protein groups. The most abundant selenoprotein group includes proteins containing Sec in the Nterminal regions, followed by an a-helix. Many of these selenoproteins exhibit thioredoxin or thioredoxin-like folds, but some proteins (e.g., MsrA) show different folds, $\mathrm{m}$ these proteins, $\mathrm{Sec}$ is the catalytic group, which often works in concert with a resolving Cys.

In the second group. Sec is located in the C-terminal sequences. These proteins so far have been described only in eukaryotes and include selenoproteins
$\mathrm{K}, \mathrm{S}, \mathrm{O}, \mathrm{I}$ and thioredoxin reductases. In thioredoxin reductases, $\mathrm{Sec}$ is the redox residue and a key component of the catalytic C-terminal Gly-Cys-Sec-Gly tetrapeptide. The function of $\mathrm{Sec}$ in other selenoproteins in this group is not known.

Selenoproteins in the third group utilize Sec to coordinate redox metals (molybdenum, tungsten and nickel) in the active sites of these proteins. This protein class includes hydrogenase, formate dehydrogenase and formylmethanofuran dehydrogenase, which are found in only in prokaryotes.

\section{Catalytic advantages and disadvantages of selenocysteine}

Sec residues serve important catalytic functions in selenoenzymes, whereas mutants in which Sec is replaced with Cys are 100-1,000 fold less active (but interestingly, these mutants do show detectable activity) [69]. m addition, comparison of catalytic functions of selenoproteins and their natural Cys-containing homologs revealed that selenoproteins often exhibit higher catalytic activities [38]. It was also found that, in some natural Cys-containing proteins, replacement of catalytic Cys residues with Sec can increase the enzyme activity [70]. All these studies are consistent with the idea that Sec provides catalytic advantages over Cys in enzyme active sites. If so, why then is Sec not used universally to maximize catalytic efficiency of thiol-based redox catalysts?

It was found that presence of Sec does not always lead to highly active catalysts. For example, Drosophila thioredoxin reductase is a Cys-containing protein, yet its activity is nearly equivalent to that of mammalian thioredoxin reductases, which are selenoproteins [71]. Instead, Sec in these proteins was proposed to provide a broader range of substrates and a broader range of microenvironmental conditions in which thioredoxin reductase activity is possible.

More recently, this question was addressed using mammalian methionine$\mathrm{R}$-sulfoxide reductases, one of which is a selenoprotein whereas the other two homologs contain Cys. It was found that Sec- and Cys-containing methionine-R-sulfoxide reductases show important differences in their catalytic mechanisms, and that having Sec has both catalytic advantages (high activity with some substrates) and disadvantages (dependence on a unique resolving Cys that assists the catalytic Sec) [34]. The use of Se should also be viewed in context of availability of selenium in the environment. Clearly, further studies are required to fully understand the reason why Sec is used or not in proteins.

\section{Selenoproteomes}

As discussed above, bioinformatics analyses allowed the identification of all or almost all selenoproteins in a variety of organisms [11]. These data about full sets of selenoproteins in organisms (selenoproteomes) are proving to be extremely useful in addressing numerous questions relevant to the biology of selenium. This information should allow, for the first time, to fully explain biological and biomedical effects of dietary selenium. This is because it is now 
possible to link individual selenoproteins or selenoprotein groups (e.g., stressrelated selenoproteins, housekeeping selenoproteins, etc.) to the specific ef fects of dietary selenium. In this respect, selenium is ahead of studies involving other trace elements (and biofactors) where new metalloproteins are still discovered biochemically and often by accident.

For example, as discussed in Chapters 13, 22-24 and 32, selenoproteins are implicated in the cancer prevention effect of selenium. However, which selenoproteins are involved is a matter of continuing investigation. Of the 25 human selenoproteins [14], several proteins may likely be excluded due to their known functions or limited expression patterns, leaving only a handful of candidate selenoproteins, which individually, or in combination, may possess cancer prevention activities.

Analyses of selenoproteomes also provide important insights into the requirement of selenium for various organisms as well as explaining Sec evolution. For example, formate dehydrogenase is present in essentially all prokaryotes that contain the machinery for Sec biosynthesis and insertion, suggesting that this protein is responsible for maintaining the Sec trait in these organisms $[15,16,59]$.

Searches of nematode selenoproteomes revealed that $C$. elegans and $C$. briggsae likely have only a single UGA codon that codes for Sec in their genomes [72]. This codon is present in the thioredoxin reductase gene, and phylogenetic analyses suggested that other selenoprotein genes were lost in these nematodes during evolution. Interestingly, fruit flies have a Cys-containing thioredoxin reductase, suggesting that this protein may compensate for the lack of selenoprotein thioredoxin reductase. Thus, it would not be surprising if an animal is identified in the future that lacks selenoproteins altogether. In turn, information about such animals (or other organisms that lost selenoproteins, such as yeast and higher plants) should help explain the changing requirements for selenium during evolution.

Recent characterization of selenoproteomes of nematodes [72], fruit flies $[12,13]$, mammals [14] and other vertebrates [52,53], and Apicomplexan parasites [55], as well as those of numerous bacteria and archaea [15,73], provided many clues in regard to the use of selenium in these organisms. Rapid progress in genome sequencing should allow application of previously developed bioinformatics tools to many additional genome projects. Recent studies show that large scale environmental genome surveys are also amenable to these applications [16]. And while the selenoprotein content of key model genomes is, in general, well characterized, the analysis of additional genomes, often uncovers novel selenoprotein families, suggesting that we only know a fraction of selenoproteins in Nature.

\section{Conclusions}

A decade ago, only several selenoproteins were known. Largely due to remarkable progress in genomic research and bioinformatics, we now have information on more than 40 selenoprotein families. In selenoproteins with known functions. Sec is a key functional group that carries out redox catalysis. Therefore, identification of each new selenoprotein provides information on the possible role of this protein in redox biology and identifies the candidate catalytic group in this protein. Importantly, this information links selenium to an ever expanding universe of biological functions dependent on this trace element. Further studies on identity and functions of selenoprotein genes should help explain known biological and biomedical effects of selenium and identify new biological processes and pathways dependent on this trace element.

\section{Acknowledgments}

This work is supported by NIH GM061603.

\section{References}

1. GL Dilworth 1982 Arch Biochem Biophys 219:30

2. VN Gladyshev, SV Khangulov, TC Stadtman 1994 Proc Natl Acad Sci USA 91:232

3. VN Gladyshev, SV Khangulov, TC Stadtman 1996 Biochemistry 35:212

4. WT Self, TC Stadtman 2000 Proc Natl Acad Sci USA 97:7208

5. D Behne, A Kyriakopoulos, H Meinhold, J Kohrle 1990 Biochem Biophys Res Commun 173:1143

6. T Tamura, TC Stadtman 1996 Proc Natl Acad Sci USA 93:1006

7. VN Gladyshev, KT Jeang, JC Wootton, DL Hatfield 1998 J Biol Chem 273:8910

8. SV Novoselov, M Rao, NV Onoshko et al 2002 EMBO J 21:3681

9. GV Kryukov, VM Kryukov, VN Gladyshev 1999 J Biol Chem 274:33888

10. A Lescure, D Gautheret, P Carbon, A Krol 1999 J Biol Chem 274:38147

11. VN Gladyshev, GV Kryukov, DE Fomenko, DL Hatfield 2004 Annu Rev Nutr 24:579

12. FJ Martin-Romero, GV Kryukov, AV Lobanov et al 2001 J Biol Chem 276:29798

13. S Castellano, N Morozova, M Morey et al 2001 EMBO Rep 2:697

14. GV Kryukov, S Castellano, SV Novoselov et al 2003 Science 300:1439

15. GV Kryukov. VN Gladyshev 2004 EMBO Rep 5:538

16. Y Zhang, DE Fomenko, VN Gladyshev 2005 Genome Biol 6:R37

17. JT Rotruck, AL Pope, HE Ganther et al 1973 Science 179:588

18. LJ Yant, Q Ran, L Rao et al 2003 Free Radic Biol Med 34:496

19. F Ursini, S Heim, M Kiess, M Maiorino, A Roveri, J Wissing, L Flohe 1999 Science 285:1393

20. VN Gladyshev, KT Jeang, TC Stadtman 1996 Proc Natl Acad Sci USA 93:6146

21. S Gromer, J Wissing, D Behne D et al 1998 Biochem J 332:591

22. QA Sun, L Kimarsky, S Sherman, VN Gladyshev 2001 Proc Natl Acad Sci USA 98:3673

23. T Sandalova, L Zhong et al 2001 Proc Natl Acad Sci USA 98:9533

24. QA Sun, Y Wu, F Zappacosta et al 1999 J Biol Chem 274:24522

25. ES Amer, A Holmgren 2000 Eur J Biochem 267:6102

26. QA Sun, F Zappacosta, V Factor et al 2001 J Biol Chem 276:3106

27. AK Rundlof, M Janard, A Miranda-Vizuete, ES Amer 2004 Free Radic Biol Med 36:64 
28. D Su, VN Gladyshev 2004 Biochemistry 43:12177

29. D Su, SV Novoselov, QA Sun et al 2005 J Biol Chem 280:26491

30. M Conrad, C Jakupoglu, SG Moreno et al $2004 \mathrm{Mol} \mathrm{Cell} \mathrm{Biol} \mathrm{24:9414}$

31. C Jakupoglu, GK Przemeck, M Schneider et al 2005 Mol Cell Biol 25:1980

32. GV Kryukov, RA Kumar, A Koc et al 2002 Proc Natl Acad Sci USA 99:4245

33. HY Kirn, VN Gladyshev $2004 \mathrm{Mol}$ Biol Cell 15:1055

34. HY Kirn, VN Gladyshev 2005 PLoS Biol 3 :e375

35. KV Korotkov, E Kumaraswamy, Y Zhou et al $2001 \mathrm{~J}$ Biol Chem 276:15330

36. E Kumaraswamy, A Malykh, KV Korotkov et al 2000 J Biol Chem 275:35540

37. AD Ferguson, VM Labunskyy, DE Fomenko et al $2006 \mathrm{~J}$ Biol Chem 281:3536

38. TC Stadtman 1996 Annu Rev Biochem 65:83

39. MJ Guimaraes, D Peterson, A Vicari et al 1996 Proc Natl Acad Sci USA 93:15086

40. RF Burk, KE Hill 2005 Annu Rev Nutr 25:215

41. GV Kryukov, VN Gladyshev 2000 Genes Cells 5:1049

42. KE Hill, J Zhou, WJ McMahan et al 2003 J Biol Chem 278:13640

43. L Schomburg, U Schweizer, B Holtmann et al 2003 Biochem J 370:397

44. SC Vendeland, MA Beilstein, JY Yeh, W Ream, PD Whanger 1995 Proc Natl Acad Sci USA 92:8749

45. KV Korotkov, SV Novoselov, DL Hatfield, VN Gladyshev 2002 Mol Cell Biol 22:1402

46. Y Ye, Y Shibata, C Yun, D Ron, TA Rapoport 2004 Nature 429:841

47. JE Curran, JB Jowett, KS Elliott et al 2005 Nat Genet 37:1234

48. B Moghadaszadeh, N Petit, C Jaillard 2001 Nat Genet 29:17

49. H Weissbach, L Resnick, N Brot N 2005 Biochim Biophys Acta 1703:203

50. SV Novoselov, M Rao. NV Onoshko et al 2002 EMBO J 21:3681

51. T Obata, Y Shiraiwa $2005 \mathrm{~J}$ Biol Chem 280:18462

52. S Castellano, SV Novoselov, GV Kryukov et al 2004 EMBO Rep 5:71

53. S Castellano, AV Lobanov et al 2005 Proc Natl Acad Sci USA 102:16188

54. SV Novoselov, D Hua, AV Lobanov, VN Gladyshev 2005 Biochem J, in press

55. AV Lobanov, CDelgado, S Rahlfs et al 2006 Nucl Acids Res 34:496

56. F Zinoni, A Birkmann, TC Stadtman, A Bock 1986 Proc Natl Acad Sci USA 83:4650

57. VN Gladyshev, SV Khangulov, MJ Axley, TC Stadtman 1994 Proc Natl Acad Sci USA 91:7708

58. JC Boyington, VN Gladyshev et al 1997 Science 275:1305

59. H Romero, Y Zhang, VN Gladyshev, G Salinas 2005 Genome Biol 6:R66

60. E Garcin, X Vemede, EC Hatchikian et al 1999 Structure 7:557

61. R Wilting, S Schorling, BC Persson, A Bock 1997 J Mol Biol 266:637

62. JA Vorholt, M Vaupel, RK Thauer 1997 Mol Microbiol 23:1033

63. GE Garcia, TC Stadtman 1991 J Bacterial 173:2093

64. M Wagner, D Sonntag, R Grimm et al 1999 Eur J Biochem 260:38

65. JR Andreesen, M Wagner, D Sonntag et al 1999 Biofactors 10:263

66. T Schrader, A Rienhofer, JR Andreesen 1999 Eur J Biochem 264:862

67. B Sohling, T Partner, KP Rucknagel, MA Wagner, JR Andreesen 2001 Biol Chem 382:979

68. DL Hatfield, VN Gladyshev 2002 Mol Cell Biol 22:3565

69. MJ Axley, A Bock, TC Stadtman 1991 Proc Natl Acad Sci USA 88:8450

70. S Hazebrouck, L Camoin, Z Faltin et al $2000 \mathrm{~J}$ Biol Chem 275:28715

71. S Gromer, L Johansson, H Bauer et al 2003 Proc Natl Acad Sci USA 100:12618

72. K Taskov, C Chappie, GV Kryukov et al 2005 Nucleic Acids Res 33:2227

73. Y Zhang, V Gladyshev, 2005 Bioinformatics 21:2580 